AperTO - Archivio Istituzionale Open Access dell'Università di Torino

Rivaroxaban use in patients with antiphospholipid syndrome and previous venous thromboembolism

This is a pre print version of the following article:

Original Citation:

Availability:

This version is available http://hdl.handle.net/2318/1609891

since 2016-11-04T15:17:44Z

Published version:

DOI:10.1097/MBC.0000000000000247

Terms of use:

Open Access

Anyone can freely access the full text of works made available as "Open Access". Works made available under a Creative Commons license can be used according to the terms and conditions of said license. Use of all other works requires consent of the right holder (author or publisher) if not exempted from copyright protection by the applicable law. 
This is the author's final version of the contribution published as:

Savino, Sciascia; Breen, Karen; Hunt, Beverley J.. Rivaroxaban use in patients with antiphospholipid syndrome and previous venous thromboembolism. BLOOD COAGULATION \& FIBRINOLYSIS. 26 (4) pp: 476-477.

DOI: 10.1097/MBC.0000000000000247

The publisher's version is available at:

http://content.wkhealth.com/linkback/openurl?sid=WKPTLP:landingpage\&an=00001721-201506000-00023

When citing, please refer to the published version.

Link to this full text:

http://hdl.handle.net/ 
Letter to the Editor

\section{Rivaroxaban use in patients with antiphospholipid syndrome and previous venous thromboembolism}

Sciascia Savino, MD, PhD Research Fellow in Thrombosis and Haemostasis, Guy's and St Thomas' Guy's \& St Thomas' Hospital, London \& Consultant Immunologist at Centro di Ricerche di Immunologia Clinica ed Immunopatologia e Documentazione su Malattie Rare (CMID), Università di Torino, Italy

Karen Breen, FRCP, FRCPath, Consultant in Thrombosis \& Thrombophilia, Guy's \& St Thomas' NHS Foundation Trust

Beverley J Hunt FRCP, FRCPath, MD, Professor of Thrombosis \& Haemostasis , Kings Health Partners \& Consultant in Thrombosis \& Thrombophilia, Guy's \& St Thomas' NHS Foundation Trust

Address for correspondence

Prof B J Hunt

Thrombosis \& Haemophilia Centre

St Thomas' Hospital

Westminster Bridge Road

London SE1 7EH

Tel 02071882736

Fax 02071882717

E mail: beverley.hunt@gstt.nhs.uk 
Patients with previous unprovoked venous thromboembolism (VTE) and persistent antiphospholipid antibodies, fulfil the criteria for antiphospholipid syndrome (APS), and are usually offered long term treatment with vitamin $\mathrm{K}$ antagonists (VKA) due to the risk of recurrent events [1].

However, VKAs require regular monitoring and maintaining the INR within the target therapeutic range can be challenging for some. Furthermore, unique to APS, VKA monitoring can be complicated by the variable responsiveness of reagents used in the INR test to lupus anticoagulants (LA), leading to instability of anticoagulation, and an inaccurate reflection of the true level of anticoagulation [2].

The world-wide approval of rivaroxaban and more latterly, other novel oral anticoagulants (NOAs), for the secondary prevention of VTE may represent a major advance for the treatment of patients with APS and previous VTE. For a NOA potentially would result in improved quality of life in patients with APS who generally require an indefinite period of anticoagulation, because unlike warfarin, NOAs do not require monitoring and have no major food or alcohol interactions and few reported drug interactions.

In order to assess rivaroxaban in comparison to VKAs in the secondary prevention of VTE in APS, the UK-based rivaroxaban in APS (RAPS trial) is currently ongoing [3]. RAPS is a prospective randomised trial of warfarin versus rivaroxaban in patients with previous VTE and APS, with or without systemic lupus erythematosus, being maintained at a target INR of 2.5 (i.e. range $2.0-3.0$ ).

In this letter, we report our preliminary experience with rivaroxaban use in patients with APS with previous VTE and poor anticoagulation control with VKA. They were started on rivaroxaban prior to the start of the RAPS study or failed to qualify for RAPS (e.g. less than 18 years old). Secondly, we aimed to evaluate the diagnostic 
accuracy of lupus anticoagulant testing in patients with APS while on rivaroxaban comparing results with their previous tests, when they were not receiving anticoagulation.

Thirty-five patients, fulfilling the current APS criteria [4] attending our Thrombosis Centre were included (24 female, 11 male, median age 47 [range 17-75], median disease duration 9 [1-18] years, median age at onset of disease 33 [15-69] years. Twenty-four had previous deep vein thrombosis, 11 had both deep vein thrombosis and pulmonary embolism. They all were receiving a VKA and had a target INR of 23, those requiring a higher target INR were excluded. The included patients' time in therapeutic range (TTR) was $65 \%$ or lower. Indication for switching from VKA to rivaroxaban $20 \mathrm{mg}$ od for secondary prevention of VTE were erratic INR control (median 13 [9-23] INR tests within the last 6 months) in 29 patients and INR constantly sub-therapeutic range in six patients.

Patients were switched from VKA to rivaroxaban $20 \mathrm{mgs}$ daily and the patients were followed for a median of 10 months [6-24]. Women in the rivaroxaban arm were advised not to become pregnant.

No further VTE, major bleeding events or serious side effects were seen except in two women there was a worsening of menorrhagia.

Extreme caution should be applied in interpreting our preliminary observations. However, we consider that NOAs might represent a promising alternative in APS patients with previous venous thromboembolism. Results from the RAPS study will be available next year and will address this issue.

Recently, Schaefer at $\mathrm{al}^{5}$. reported the failure of dabigatran and rivaroxaban for secondary thromboprophylaxis in three patients with APS. Our results disagree with 
their experience, probably due to the different subset of patients in the two studies. Two of three patients reported by Schaefer at al [5] had previous arterial thrombotic events (cerebral infarction and radial artery thrombosis). Previous arterial thrombosis was an exclusion criterion in our study, as we use an INR target of 3-4 in these patients when compared to patient with previous VTE. Therefore, until the equivalent dosing of NOAs to an INR of 3-4 is known, we will not be using NOAs for secondary thromboprophylaxis in patients with previous arterial event. Patient three was reported as having cirrhosis and oesophageal varices. It is arguable to attribute the portal vein thrombosis to the failure of rivaroxaban only, as several additional factors might have contributed. Firstly, portal vein thrombosis is a common complication of liver cirrhosis [6]. Secondly, the sub-acute/chronic features shown at the venogram might call into questioning the timing of the event. Lastly, rivaroxaban was stopped for six days before admission.

It is debatable if the detection of LA may be affected by rivaroxaban. We compared testing for LA in patients on rivaroxaban with their previous tests, when they were not receiving anticoagulation. Diagnostic accuracy for LA testing during rivaroxaban treatment is shown in Table 1. Taipan snake venom time (TSVT) had the best specificity and positive predictive value when compared to dilute activated partial thromboplastin time (dAPPT) and dilute Russell's viper venom time (dRVVT). Our results support previous observations suggesting that Taipan/Ecarin time is poorly affected by the presence of rivaroxaban [7]. The observation that dRVVT is the test most affected by rivaroxaban [8] is not surprising, as Russell's Viper Venom and rivaroxaban share the same target (factor $\mathrm{Xa}$ ). However, in our study false positive results were seen with all types of LA testing, suggesting they cannot be used 
diagnostically while patients receive rivaroxaban; although this needs confirming in studies design specifically to address this question.

In summary our study suggests the use of rivaroxaban therapy for secondary thromboprophylaxis for APS patients with previous VTE looks promising, but that LA testing in patients receiving rivaroxaban seems unreliable. In the interim, our observations suggest that rivaroxaban may be considered cautiously as an alternative anticoagulant in APS patients with previous VTE, who require a target INR of 2-3, but have poor anticoagulant control with VKA. 
Acknowledgment

SS, KR and BJH designed the study, analyzed the data and wrote the paper.

\section{Authorship}

The authors declare no conflict of interest and declare: no support from any organisation for the submitted work; no financial relationships with any organisations that might have an interest in the submitted work in the previous 3 years; no other relationships or activities that could appear to have influenced the submitted work. 


\section{References}

1. Ruiz-Irastorza G, Crowther M, Branch W, Khamashta MA Antiphospholipid syndrome. Lancet. 2010;376:1498-509.

2. Tripodi A, Chantarangkul V, Clerici M, Negri B, Galli M, Mannucci PM.. Laboratory control of oral anticoagulant treatment by the INR system in patients with the antiphospholipid syndrome and lupus anticoagulant. Results of a collaborative study involving nine commercial thromboplastins. $\mathrm{Br} \mathrm{J}$ Haematol. 2001;115:672-8.

3. Rivaroxaban in AntiPhospholipid Syndrome, IRSCTN 68222801. http://www.controlled-trials.com/ISRCTN68222801. Last accessed 25th July 2014.

4. Miyakis S1, Lockshin MD, Atsumi T, Branch DW, Brey RL, Cervera R, et al. International consensus statement on an update of the classification criteria for definite antiphospholipid syndrome (APS). J Thromb Haemost. 2006;4:295-306.

5. Schaefer JK, McBane RD, Black DF, Williams LN, Moder KG, Wysokinski WE. Failure of dabigatran and rivaroxaban to prevent thromboembolism in antiphospholipid syndrome: a case series of three patients. Thromb Haemost. 2014;112(5).

6. Qi X, Han G, Fan D. Management of portal vein thrombosis in liver cirrhosis. Nat Rev Gastroenterol Hepatol. 2014;11:435-46

7. van Os GM, de Laat B, Kamphuisen PW, Meijers JC, de Groot PG. Detection of lupus anticoagulants in the presence of rivaroxaban using Taipan snake venom time. J Thromb Haemost. 2011;9:1657-9. 
8. Merriman E, Kaplan Z, Butler J, Malan E, Gan E, Tran H. Rivaroxaban and false positive lupus anticoagulant testing. Thromb Haemost. 2012;105:385-6 
Table 1

Diagnostic accuracy of lupus anticoagulant testing in patients with APS while on rivaroxaban comparing results with their previous tests, when they were not receiving anticoagulation.

\begin{tabular}{|c|c|c|c|c|}
\hline & Sensitivity (\%) & Specificity (\%) & NPP (\%) & PPV (\%) \\
\hline dRVVT* & 94 & 50 & 88 & 69 \\
\hline dAPPT* & 84 & 75 & 80 & \\
& & & & 91 \\
\hline TSVT & 58 & 93 & 65 & \\
\hline
\end{tabular}

dAPPT, dilute activated partial thromboplastin time; dRVVT, Dilute Russell's viper venom time; TSVT, Taipan snake venom time; Negative predictive value, NPP, positive predictive value; PPV; results were not available in 3 patients. *includes screening, mixing and confirmation stages. 